\title{
miR-124-3p regulates angiogenesis in peripheral arterial disease by targeting STAT3
}

\author{
YEFEI SHI ${ }^{1 *}$, XU XU $^{1 *}$, PEIPEI LUAN ${ }^{1}$, WENXIN KOU ${ }^{1}$, MINGJIE $^{2}{ }^{2}$, QING YU $^{1}$, \\ JIANHUI ZHUANG ${ }^{1}$, YAWEI XU ${ }^{1}$, WENHUI PENG ${ }^{1}$ and WEIXIA JIAN ${ }^{2}$
}

\author{
${ }^{1}$ Department of Cardiology, Shanghai Tenth People's Hospital, Tongji University School of Medicine, \\ Shanghai 200072; ${ }^{2}$ Department of Endocrinology, Xinhua Hospital Affiliated to Shanghai Jiaotong University, \\ School of Medicine, Shanghai 200092, P.R. China
}

Received February 12, 2020; Accepted September 9, 2020

DOI: $10.3892 / \mathrm{mmr} .2020 .11538$

\begin{abstract}
Peripheral arterial disease (PAD) is the third leading cause of cardiovascular morbidity worldwide, after coronary artery disease and stroke. As endogenous regulators of gene expression, microRNAs (miRs) are implicated in the development and progression of various diseases, including types of cancer, autoimmune diseases and heart diseases. In the present study, the role of miR-124-3p in PAD was investigated. The reverse transcription-quantitative PCR results indicated that the expression levels of miR-124-3p were significantly increased in the ischemic tissue of the hindlimb ischemia (HLI) model and in hypoxic human umbilical vein endothelial cells compared with the corresponding control groups. Proliferation, wound healing and tube formation assays demonstrated the inhibition of miR-124-3p on angiogenesis in vitro and the HLI model indicated the same function of miR-124-3p in vivo. A dual-luciferase reporter revealed STAT3 as the target of miR-124-3p. The expression levels of miR-124-3p in human blood were negatively correlated with ankle-brachial index, which is an index for the evaluation of the severity of PAD. Collectively, the present study indicated that miR-124-3p was a critical regulator of angiogenesis in PAD, and a potential diagnostic, prognostic and therapeutic target for PAD.
\end{abstract}

Correspondence to: Professor Wenhui Peng, Department of Cardiology, Shanghai Tenth People's Hospital, Tongji University School of Medicine, 301 Middle Yanchang Road, Shanghai 200072, P.R. China

E-mail: pwenhui@tongji.edu.cn

Professor Weixia Jian, Department of Endocrinology, Xinhua Hospital Affiliated to Shanghai Jiaotong University, School of Medicine, 1665 Kongjiang Road, Shanghai 200092, P.R. China

E-mail: jianweixia@xinhua.com.cn

*Contributed equally

Key words: microRNA-124-3p, peripheral arterial disease, hindlimb ischemia, angiogenesis, STAT3

\section{Introduction}

Peripheral arterial disease (PAD) is a common peripheral circulatory problem, in which narrowed arteries occur, especially in the lower extremities (1,2). PAD has been reported as a highly age-related vascular disease, typically occurring in patients aged 60-70 years, which leads to health problems (3). Surgical and catheter-based revascularization that target primary occluded macro vessels are the primary conventional treatment strategies for PAD. Unfortunately, these high-cost methods result in limited improvement in the microcirculation, due to complications that occur in patients with PAD (4). Angiogenesis is a process of the endothelium, by which a new vessel is formed from the old one (5). Therapeutic angiogenesis has been studied for two decades, focusing on the overexpression of growth factors, which have been identified as factors that evoke angiogenesis in vitro in $\mathrm{PAD}$, including vascular endothelial growth factor (VEGF) and hepatocyte growth factor $(6,7)$. However, almost none of the trials met the expected goal, including significant clinical remission, which has led researchers to investigate other molecules that are responsible for the limited effects.

MicroRNAs (miRNAs/miRs), a class of 22 nucleotide-long, small non-coding RNAs, regulate gene expression by repressing post-transcription to modulate cell fate decisions $(8,9)$. miRNAs were first defined as endogenous regulators for a variety of cellular physiologic and pathologic processes (10). To date, 2,654 mature human miRNAs have been identified (11). Recently, certain miRNAs have been identified to be associated with ischemia-reperfusion injury $(12,13)$. A number of identified miRNAs have been reported to serve as endogenous negative regulators of angiogenesis. For example, the antiangiogenesis function of miR-92a has been verified in vitro and in vivo $(14,15)$. However, whether other miRNAs serve the same role as miR-92a is not completely understood. miR-124-3p has been reported to regulate glioma angiogenesis $(16,17)$ and participate in ischemic diseases $(18,19)$. Nevertheless, there are limited studies on the role of miR-124-3p in PAD. Therefore, the present study investigated the effect of miR-124-3p on endothelial cells (ECs) and PAD.

In the present study, the role of miR-124-3p in PAD was investigated in the blood of patients with PAD, as well as in 
the ECs and in the hindlimb ischemia model. In addition, the target of miR-124-3p was identified in the present study.

\section{Materials and methods}

Cell culture, stimulation and transfection. Human umbilical vein ECs (HUVECs; cat. no. 8000; ScienCell Research Laboratories, Inc.) were cultured and stimulated at $37^{\circ} \mathrm{C}$ in 5\% $\mathrm{CO}_{2}$ in Endothelial Cell Medium (cat. no. 1001; ScienCell Research Laboratories, Inc.) supplemented with 5\% FBS (cat. no. 0025; ScienCell Research Laboratories, Inc.), 1\% endothelial cell growth supplement (cat. no. 1052; ScienCell Research Laboratories, Inc.) and 1\% penicillin/streptomycin solution (cat. no. 0503; ScienCell Research Laboratories, Inc.). Cobalt (II) chloride hexahydrate (500 $\mu \mathrm{M}$, cat. no. C8661; Sigma-Aldrich; Merck KGaA) was used to stimulate HUVECs at $37^{\circ} \mathrm{C}$ in $5 \% \mathrm{CO}_{2}$ for 24 or $48 \mathrm{~h}$ when cells reach $90 \%$ confluence. At $\sim 80 \%$ confluence, HUVECs were transfected with mimic (50 nM; Guangzhou RiboBio Co., Ltd.), inhibitor (100 nM; Guangzhou RiboBio Co., Ltd.), small interfering (si)RNA ( $25 \mathrm{nM}$; Shanghai GenePharma Co., Ltd.) or corresponding negative controls (NCs, $25 \mathrm{nM}$; Shanghai GenePharma Co., Ltd.) for $36 \mathrm{~h}$ using Lipofectamine ${ }^{\circledR} 2000$ (cat. no. 11668019; Invitrogen; Thermo Fisher Scientific, Inc.) according to the manufacturer's instructions. The sequences of the mimics, inhibitors and siRNAs were as follows: NC mimic forward, 5'-UUUGUACUACACAAAAGUACUG-3' and reverse, 5'-CAGUACUUUUGUGUAGUACAAA-3'; miR-124-3p mimic forward, 5'-UAAGGCACGCGGUGA AUGCC-3' and reverse, 5'-GGCAUUCACCGCGUGCCU UA-3'; NC inhibitor, 5'-CAGUACUUUUGUGUAGUACA AA-3'; miR-124-3p inhibitor, 5'-GGCAUUCACCGCGUG CCUUA-3'; si-NC forward, 5'-UUCUCCGAACGUGUC ACGU-3' and reverse, 5'-ACGUGACACGUUCGGAGAA-3'; si-STAT3 forward, 5'-CCCGGAAAUUUAACAUUCU-3' and reverse, 5'-AGAAUGUUAAAUUUCCGGG-3'.

$R N A$ isolation and reverse transcription-quantitative PCR $(R T-q P C R)$. Total RNA was isolated from human blood, HUVECs, gastrocnemius and the blood of HLI model mice using TRIzol ${ }^{\circledR}$ (cat. no. 15596026; Invitrogen; Thermo Fisher Scientific, Inc.) according to the manufacturer's instructions. Total RNA $(1 \mu \mathrm{g})$ was reverse transcribed into cDNA using the PrimeScript RT Reagent kit (cat. no. RR037A; Takara Bio, Inc.) as follows: $37^{\circ} \mathrm{C}$ for $15 \mathrm{~min}, 85^{\circ} \mathrm{C}$ for $5 \mathrm{sec}$ and $4^{\circ} \mathrm{C}$ thereafter. Subsequently, qPCR was performed using KAPA SYBR FAST qPCR Master Mix (cat. no. KM4101; Roche Diagnostics) as follows: $95^{\circ} \mathrm{C}$ for $3 \mathrm{~min}$, then 40 cycles of $95^{\circ} \mathrm{C}$ for $10 \mathrm{sec}$ and $60^{\circ} \mathrm{C}$ for $30 \mathrm{sec}$. miRNA and mRNA expression levels were quantified using the $2^{-\Delta \Delta \mathrm{Cq}}$ method (20) and normalized to the internal reference genes U6 and GAPDH, respectively. Stem-loop primers of miR-124-3p and U6 were purchased from Guangzhou RiboBio Co., Ltd. The primer sequences of miR-124-3p, U6, STAT3 and GAPDH were as follows: miR-124-3p forward, 5'-TCTTTAAGGCACGCG GTG-3' and reverse, TATGGTTTTGACGACTGTGTGAT; U6 forward, 5'-CTCGCTTCGGCAGCACA-3' and reverse, 5'-AACGCTTCACGAATTTGCGT-3'; STAT3 forward, 5'-CAGCAGCTTGACACACGGTA-3' and reverse, 5'-AAA CACCAAAGTGGCATGTGA-3'; and GAPDH forward,
5'-GGAGCGAGATCCCTCCAAAAT-3' and reverse, 5'-GGC TGTTGTCATACTTCTCATGG-3'.

Proliferation assay. Cell proliferation was determined using the Edu Cell Proliferation kit (cat. no. C10339; Invitrogen; Thermo Fisher Scientific, Inc.). Following 12 h serum starvation, HUVECs were incubated with Edu-labeling mixture $(10 \mu \mathrm{M})$ in combination with recombinant human VEGFA-165 (50 ng/ml; cat. no. $100-20$; PeproTech, Inc.) for $12 \mathrm{~h}$ at $37^{\circ} \mathrm{C}$ in $5 \% \mathrm{CO}_{2}$. Images (four pictures of each group) were captured by an Olympus IX83 fluorescence microscope (Olympus Corporation) at 10x magnification. The rate of cell proliferation was calculated using the following formula: Number of $\mathrm{Edu}^{+}$cells/total number of cells in each field.

Wound healing assay. At $\sim 90 \%$ confluence, the limit of HUVEC proliferation, a scratch was made in the center of each well using the tip of a $200 \mu 1$ pipette. Subsequently, serum-starved HUVECs were cultured with VEGFA-165 $(50 \mathrm{ng} / \mathrm{ml})$. Images of the wounds were captured at 0 and $24 \mathrm{~h}$ by an Olympus CKX53 inverted microscope at 4x magnification (Olympus Corporation). Cell migration was analyzed using ImageJ software (version 1.52a; National Institutes of Health).

Tube formation assay. For the assay, 96-well plates were pre-coated with Matrigel (cat. no. 354230; Corning, Inc.) at $37^{\circ} \mathrm{C}$ for $30 \mathrm{~min}$, and then HUVECs were seeded $\left(2 \times 10^{4}\right.$ cells/well $)$ into the Matrigel. Following culture with VEGFA-165 $(50 \mathrm{ng} / \mathrm{ml})$ for $8 \mathrm{~h}$ at $37^{\circ} \mathrm{C}$ in $5 \% \mathrm{CO}_{2}$, images were captured to detect tube formation by an Olympus CKX53 inverted microscope at 4x magnification (Olympus Corporation). The total tube length was assessed using ImageJ software (version 1.52a; National Institutes of Health).

Dual-luciferase reporter assay. The target of miR-124-3p was predicted using TargetScan (version 7.1, http://www. targetscan.org). The 3'-untranslated region (3'-UTR) luciferase reporter construct of STAT3 was cloned downstream of the Renilla luciferase gene in the pSI-check2 vector (Hanbio Biotechnology Co., Ltd.). 293T cells (cat. no. CRL-11268; American Type Culture Collection) were seeded into a 96-well plate with density of $80 \%$. Subsequently, 293T cells were co-transfected with 5 pmol miR-124-3p mimic or NC mimic and $0.16 \mu \mathrm{g}$ STAT3-wild-type (WT) or STAT3-mutant (Mut) using Lipofectamine ${ }^{\circledR} 2000$ (cat. no. 11668019; Invitrogen; Thermo Fisher Scientific, Inc.). At 48 h post-transfection, luciferase activities were detected using the Dual-Luciferase Reporter Assay System (cat. no. E1910; Promega Corporation). Firefly luciferase activities were normalized to Renilla luciferase activities.

Protein extraction and western blotting. Total protein was extracted from HUVECs using Cell Lysis Buffer (cat. no. 9803s; Cell Signaling Technology, Inc.) with protease inhibitors (cat. no. 04693159001; Roche Diagnostics). The bicinchoninic acid method was used for protein determination. Protein $(10 \mu \mathrm{g})$ was separated via $8 \%$ SDS-PAGE and transferred to PVDF membranes, which were blocked with $5 \%$ bovine serum albumin at room temperature for $1 \mathrm{~h}$. 
Subsequently, the membranes were incubated overnight at $4^{\circ} \mathrm{C}$ with the following primary antibodies: STAT3 $(1: 1,000$; cat. no. 9139; Cell Signaling Technology, Inc.) and GAPDH (1:5,000; cat. no. 60004-1-Ig; ProteinTech Group, Inc.). Following primary incubation, the membranes were incubated with horseradish peroxidase-conjugated secondary antibodies (anti-mouse IgG, 1:2,000, cat. no. ab205719, Abcam) at room temperature for $1 \mathrm{~h}$. Protein bands were visualized using chemiluminescence (cat. no. 180-5001; Tanon Science and Technology Co., Ltd.) and detected using the Amersham Imager 600 system (GE Healthcare Life Sciences). Protein expression was semi-quantified using ImageJ software (version 1.52a; National Institutes of Health) with GAPDH as the loading control.

HLI model and detection of perfusion recovery. A total of 50 eight-week-old male mice (18 to $22 \mathrm{~g}$ ) were used for HLI model. The HLI model was established as previously described (21). Briefly, mice were anesthetized with an intraperitoneal injection of ketamine $(80 \mathrm{mg} / \mathrm{kg})$ and xylazine $(5 \mathrm{mg} / \mathrm{kg})$. Excision and ligation were performed on the left femoral artery. For the sham operation, excision was performed on the contralateral hindlimb. miR-124-3p was overexpressed using agomiR-124-3p and inhibited using antagomiR-124-3p. Accordingly, mice were injected with 5 nmol agomiR-124-3p or agomiR-NC (Guangzhou RiboBio Co., Ltd.) into two sites of the gastrocnemius and one site of the tibialis anterior muscle on day 0,7 and 14 post-HLI. In addition, $8 \mathrm{mg} / \mathrm{kg}$ antagomiR-124-3p or antagomiR-NC (Guangzhou RiboBio Co., Ltd.) were injected via a tail vein injection on day 0,7 and 14 post-HLI. The method was adapted from Hazarika et al (22). Perfusion recovery was detected via laser Doppler imaging (Moor Instruments Ltd.) on day 0, 3 , 7, 14 and 21 post-HLI and quantified using moorLDI Image Processing software (version 5.3; Moor Instruments Ltd.). Perfusion in the ischemic limb was normalized to the sham limb for each mouse. The mice were anesthetized by isoflurane $(5 \%)$ inhalation and euthanized by $\mathrm{CO}_{2}(100 \%)$ inhalation at a rate of $30 \%$ volume/minute. Animal studies were conducted in compliance with the Guide for the Care and Use of Laboratory Animals published by the NIH (23) and approved by the Animal Care and Use Committees of the Shanghai Tenth People's Hospital (approval no. SHDSYY-2019-2149).

The agomir and antagomir sequences were as follows: agomiR-NC forward, 5'-UUCUCCGAACGUGUCACG U3' and reverse, 5'-ACGUGACACGUUCGGAGAA-3'; agomiR-124-3p forward, 5'-UAAGGCACGCGGUGAAUG CC-3' and reverse, 5'-GGCAUUCACCGCGUGCCUUA-3'; antagomiR-NC, 5'-CAGUACUUUUGUGUAGUACAA-3'; and antagomiR-124-3p, 5'-GGCAUUCACCGCGUGCCU UA-3'.

Immunofluorescence. Gastrocnemius tissues of mice were harvested and processed in optimal cutting temperature compound (cat.no. 4583, Sakura Finetek USA, Inc.). The treated tissues were sliced into $5 \mu \mathrm{m}$-thick sections. Subsequently, sections were fixed in $4 \%$ formaldehyde for $15 \mathrm{~min}$, incubated in $10 \%$ normal goat serum (cat. no. AR0009, Wuhan Boster Biological Technology, Ltd.) and $0.5 \%$ Triton X-100 for $30 \mathrm{~min}$, all at room temperature. The sections were incubated with a rat anti-mouse CD31 antibody (1:100; cat. no. 557355; BD Biosciences) overnight at $4^{\circ} \mathrm{C}$. Following washing with PBS, the sections were incubated with a secondary antibody (Alexa Fluor 594-conjugated donkey anti-rat, 1:200, cat. no. 34412ES60; Shanghai Yeasen Biotechnology Co., Ltd.) at $37^{\circ} \mathrm{C}$ for $1 \mathrm{~h}$ in the dark. Nuclear staining was performed using 4,6-amidine-2-phenylindoles (cat. no. 28718-90-3; Merck $\mathrm{KGaA}$ ) for $30 \mathrm{~min}$ at room temperature. Fluorescence images were obtained using an Olympus IX83 fluorescence microscope at 10x magnification (Olympus Corporation) and quantified using ImageJ software (version 1.52a; National Institutes of Health).

Study population. A total of 49 patients with PAD and 47 sexand age-matched participants without PAD were recruited at the Department of Endocrinology, Xinhua Hospital Affiliated to Shanghai Jiaotong University School of Medicine and Department of Cardiology, Shanghai Tenth People's Hospital between August 2019 and July 2020. According to the 2016 AHA/ACC guidelines on PAD, ankle-brachial index (ABI) was used to diagnose and evaluate the severity of PAD. PAD was diagnosed in patients with an ABI <0.9 (24), which was measured and calculated according to the method described by Aboyans et al (25). Patients with cancer, acute myocardial infarction, severe kidney failure, acute infection or connective tissue disease were excluded from the present study. It is recognized that patients with hypertension or diabetes are at higher risk of PAD $(3,4)$. Hypertension was defined as systolic or diastolic blood pressure $\geq 140 / 90 \mathrm{mmHg}$, or normal blood pressure following the admission of antihypertensive medications prior to recruitment. Diabetes was defined as a fasting blood glucose $\geq 7 \mathrm{mmol} / \mathrm{l}$, non-fasting plasma glucose level of $\geq 11.1 \mathrm{mmol} / 1$ or normal blood glucose levels following known treatment for diabetes. Blood samples $(4 \mathrm{ml})$ were collected from participants at the time of admission. Written informed consent was obtained from all patients. The present study was approved by the Ethics Committee of the Shanghai Tenth People's Hospital (approval no. 2019-K-153).

Statistical analysis. Data are presented as the mean \pm standard deviation. Comparisons between two groups were analyzed using the unpaired Student's t-test. Comparisons among multiple groups were analyzed using one-way or two-way ANOVA followed by Bonferroni's post hoc test. The parameters from baseline characteristics of patients with PAD and non-PAD individuals were analyzed using the $\chi^{2}$ test (age, BMI and blood lipid were excluded). Pearson's correlation analysis was performed to investigated the correction between ABI and the expression level of miR-124-3p. Statistical analyses were performed using GraphPad Prism software (version 6.01; GraphPad Software, Inc.). $\mathrm{P}<0.05$ was considered to indicate a statistically significant difference. All experiments were repeated $\geq 3$ times.

\section{Results}

Expression levels of miR-124-3p are increased in the HLI model and hypoxic HUVECs. To identify the role of miR-124-3p in the HLI model, the dynamic expression of miR-124-3p was detected in the ligated ischemic gastrocnemius. Total 

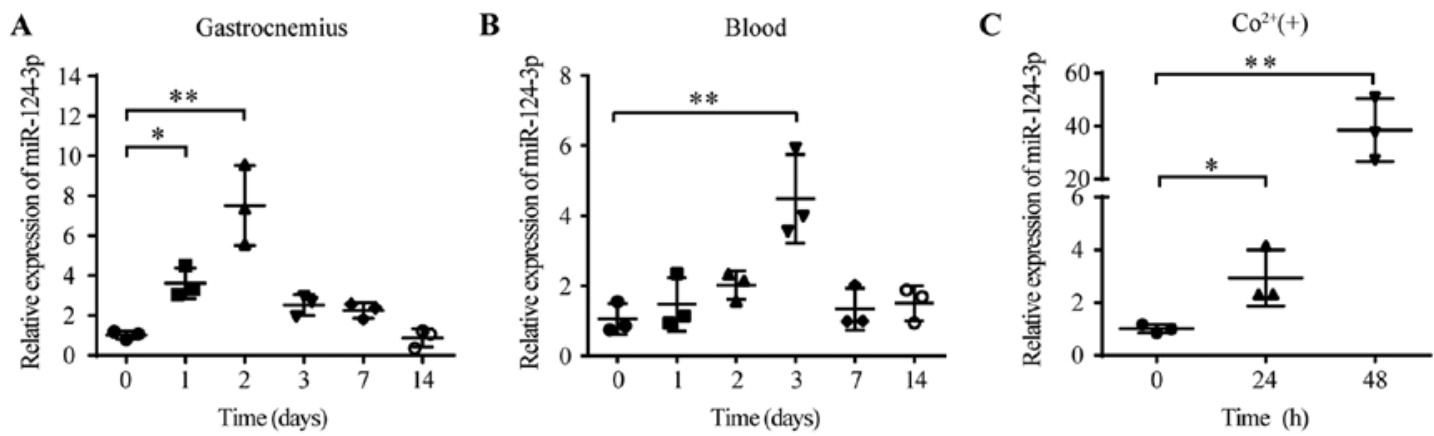

Figure 1. Expression levels of miR-124-3p are increased in the HLI model and hypoxic HUVECs. Relative expression of miR-124-3p in the (A) gastrocnemius and (B) blood of HLI model on days 0,1,2,3,7 and 14. (C) Cobalt-induced relative expression of miR-124-3p in HUVECs under hypoxic conditions (500 $\mu \mathrm{M})$ for 24 and $48 \mathrm{~h}$. The experiments were repeated three times. ${ }^{*} \mathrm{P}<0.05$ and ${ }^{* *} \mathrm{P}<0.01$. miR, microRNA; HLI, hindlimb ischemia; HUVECs, human umbilical vein endothelial cells.

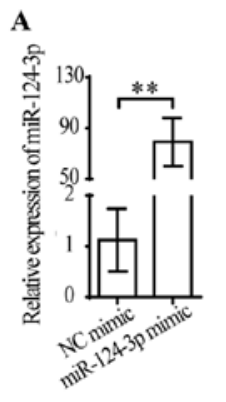

D

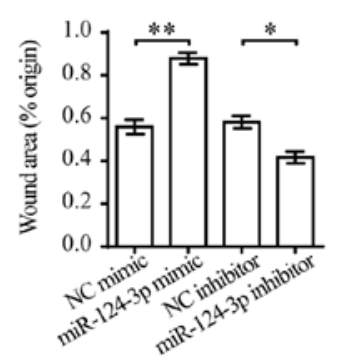

B

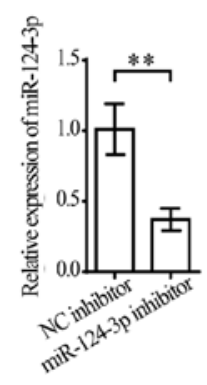

E

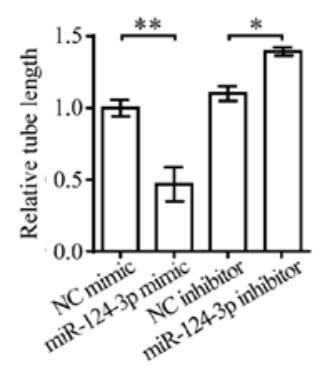

C

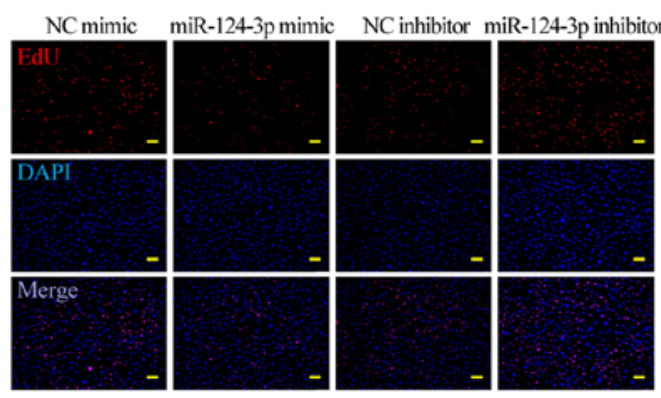

F NC mimic miR-124-3p mimic NC inhibitor miR-124-3p inhibitor

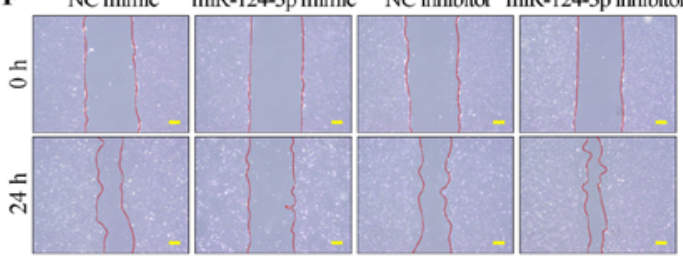

G

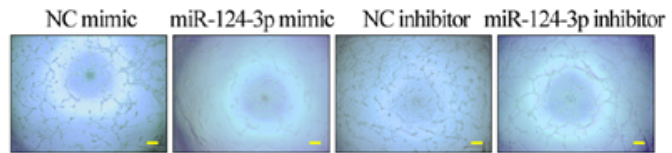

Figure 2. miR-124-3p impairs the functions of VEGFA-165-treated HUVECs. (A) miR-124-3p expression in miR-124-3p mimic-transfected HUVECs. (B) Effect of miR-124-3p on HUVEC proliferation following culture with VEGFA-165 for $12 \mathrm{~h}$. (C) Representative images of the EdU staining assay. Scale bar, $100 \mu \mathrm{m}$. Effect of miR-124-3p on HUVEC (D) migration and (E) tube formation following culture with VEGFA-165 for 0 or $24 \mathrm{~h}$. Representative images of the (F) wound healing and (G) tube formation assays. Scale bar, $100 \mu \mathrm{m}$. "P<0.05 and ${ }^{* *} \mathrm{P}<0.01$. miR, microRNA; VEGFA, vascular endothelial growth factor-A; HUVECs, human umbilical vein endothelial cells; NC, negative control.

RNA was extracted from the gastrocnemius and blood of HLI model mice. The RT-qPCR results suggested that the levels of miR-124-3p in the ischemic gastrocnemius were significantly upregulated on day 2 compared with day 0 (day 2 , $7.50 \pm 2.00$ vs. day $0,1.01 \pm 0.20$; Fig. 1A). However, the levels of miR-124-3p in the blood peaked on day 3 (day $3,4.48 \pm 1.26 \mathrm{vs}$. day $0,1.05 \pm 0.43$; Fig. 1B). Hypoxia-inducible factor- $\alpha$ (HIF- $\alpha$ ) is a powerful inducer of angiogenesis $(26,27)$. Cobalt has been reported to mimic hypoxia by preventing the degradation of HIF- $\alpha$ (28). Therefore, cobalt was used to mimic hypoxia in HUVECs. The levels of miR-124-3p were significantly increased following cobalt-induced hypoxia $(500 \mu \mathrm{M})$ at $48 \mathrm{~h}$ compared with $0 \mathrm{~h}(48 \mathrm{~h}, 38.54 \pm 11.88$ vs. $0 \mathrm{~h}, 1.01 \pm 0.16$; Fig. 1C). Collectively, the results indicated that miR-124-3p levels were increased in HUVECs and tissues under hypoxic conditions, which suggested that miR-124-3p might be essential for the progression of HLI.

miR-124-3p impairs the functions of VEGFA-165-treated HUVECs. VEGFA-165-stimulated ECs display enhanced proliferation, migration and stability for angiogenesis (29). Moreover, the aforementioned results provided evidence for investigating the functions of miR-124-3p in ECs (Fig. 1C). miR-124-3p mimic was used to overexpress miR-124-3p, whereas miR-124-3p inhibitor was used to knock down miR-124-3p. At $48 \mathrm{~h}$ post-transfection, the expression levels of miR-124-3p were measured via RT-qPCR. Compared with the corresponding NCs, miR-124-3p was successfully overexpressed by mimic and knocked down by inhibitor in HUVECs (Fig. 2A). To investigate the effect of miR-124-3p mimic and 
Table I. Baseline characteristics of patients with PAD and non-PAD individuals.

\begin{tabular}{lccr}
\hline Variable & Non-PAD $(\mathrm{n}=47)$ & PAD $(\mathrm{n}=49)$ & P-value \\
\hline Age $($ years $)$ & $62.60 \pm 11.88$ & $65.90 \pm 8.00$ & 0.104 \\
Gender $(\mathrm{male})$ & $22(46.81 \%)$ & $29(59.18 \%)$ & 0.225 \\
BMI $\left(\mathrm{kg} / \mathrm{m}^{2}\right)$ & $24.16 \pm 2.87$ & $24.28 \pm 1.87$ & 0.801 \\
Smoking & $11(23.40 \%)$ & $28(57.14 \%)$ & 0.001 \\
Blood lipid & & & \\
Total cholesterol $(\mathrm{mmol} / \mathrm{l})$ & $4.22 \pm 1.02$ & $4.70 \pm 1.45$ & 0.059 \\
Triglyceride (mmol/l) & $1.99 \pm 0.84$ & $1.70 \pm 0.87$ & 0.092 \\
High-density lipoprotein $(\mathrm{mmol} / \mathrm{l})$ & $1.21 \pm 0.27$ & $1.43 \pm 0.50$ & 0.008 \\
Low-density lipoprotein $(\mathrm{mmol} / \mathrm{l})$ & $2.74 \pm 0.86$ & $2.89 \pm 0.98$ & 0.413 \\
Medical history & & & \\
Coronary heart disease & $9(19.14 \%)$ & $24(48.98 \%)$ & 0.003 \\
Diabetes & $31(65.96 \%)$ & $46(93.88 \%)$ & 0.001 \\
Hypertension & $24(51.06 \%)$ & $33(67.35 \%)$ & 0.146 \\
Statins & $18(38.30 \%)$ & $28(57.14 \%)$ & 0.071 \\
\hline
\end{tabular}

Data are presented as the mean \pm SD or n $(\%)$. Age, BMI and blood lipid variables were analyzed using the Student's t-test. All other parameters were analyzed using the $\chi^{2}$ test. PAD, peripheral arterial disease; BMI, body mass index.

inhibitor, alterations to the cell phenotype were examined. miR-124-3p mimic significantly inhibited HUVEC proliferation compared with NC mimic, whereas miR-124-3p inhibitor significantly enhanced HUVEC proliferation compared with NC inhibitor (Fig. 2B and C). Subsequently, the effect of miR-124-3p on HUVEC migration was analyzed. The wound healing assay results indicated that HUVEC migration was significantly inhibited by miR-124-3p mimic compared with NC mimic, but significantly enhanced by miR-124-3p inhibitor compared with NC inhibitor (Fig. 2D and F). The tube formation assay was conducted to evaluate the effect of miR-124-3p on angiogenesis. miR-124-3p mimic significantly inhibited tube formation compared with NC mimic, whereas miR-124-3p inhibitor significantly enhanced tube formation compared with $\mathrm{NC}$ inhibitor (Fig. 2E and G). Therefore, the results suggested that miR-124-3p impaired HUVEC functions in vitro.

STAT3 is a target of miR-124-3p. TargetScan indicated that STAT3 was a potential target of miR-124-3p (Fig. 3A). STAT3 is a member of the STAT protein family, and emerging evidence has suggested that it is a regulator of angiogenesis (30). To verify STAT3 as a target of miR-124-3p, luciferase reporter plasmids containing miR-124-3p binding sites in the 3'-UTRs of STAT3 were constructed. The dual-luciferase reporter assay results indicated that miR-124-3p mimic significantly decreased the luciferase activities of STAT3-WT compared with NC mimic (Fig. 3C). Furthermore, the protein expression levels of STAT3 were measured via western blotting. STAT3 protein expression levels were significantly decreased in the miR-124-3p mimic group compared with the NC mimic group, but significantly increased in the miR-124-3p inhibitor group compared with the NC inhibitor group (Fig. 3B and D). To further investigate the effect of miR-124-3p on STAT3, a rescue experiment was conducted. In brief, STAT3 knockdown was performed in combination with miR-124-3p inhibitor transfection in
HUVECs. Subsequently, proliferation, wound healing and tube formation assays were preformed to assess angiogenesis in HUVECs. The RT-qPCR results indicated that STAT3 mRNA expression levels were significantly decreased by si-STAT3 compared with si-NC (Fig. 3E). Moreover, STAT3 knockdown significantly suppressed EC proliferation (Fig. 3F and G), migration (Fig. 3H and I) and tube formation (Fig. 3J and K) compared with the NC group. miR-124-3p inhibitor reversed STAT3 knockdown-mediated inhibition of angiogenesis in ECs. Collectively, the results indicated that miR-124-3p regulated the functions of HUVECs by targeting STAT3.

miR-124-3p impairs perfusion recovery and capillary density in the HLI model. To investigate whether miR-124-3p directly modulated perfusion recovery following HLI, agomiR-124-3p and antagomiR-124-3p were injected in femoral artery-ligated mice. The results suggested that agomiR-124-3p-treated mice displayed significantly impaired perfusion recovery from day 14 post-HLI compared with agomiR-NC-treated mice (Fig. 4A and B). By contrast, antagomiR-124-3p significantly improved perfusion recovery from day 14 post-HLI compared with antagomiR-NC (Fig.4A and C). Consistent with blood perfusion recovery, capillary density of ischemic muscles displayed the same tendency. AgomiR-124-3p-treated mice displayed significantly fewer $\mathrm{CD} 31^{+}$cells in the gastrocnemius compared with agomiR-NC-treated mice, which suggested that agomiR-124-3p might inhibit angiogenesis in ischemic hindlimbs. By contrast, antagomiR-124-3p significantly increased the number of $\mathrm{CD} 31^{+}$ cells compared with antagomiR-NC, suggesting activation of angiogenesis in gastrocnemius (Fig. 4D and E). In combination, the results indicated that miR-124-3p regulated angiogenesis following ischemic injury in mouse hindlimbs.

miR-124-3p expression is increased in patients with PAD. The aforementioned results indicated that the expression of 
A

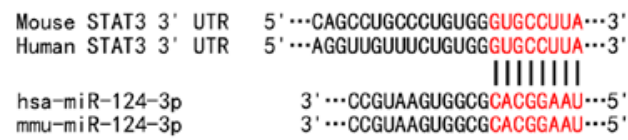

C
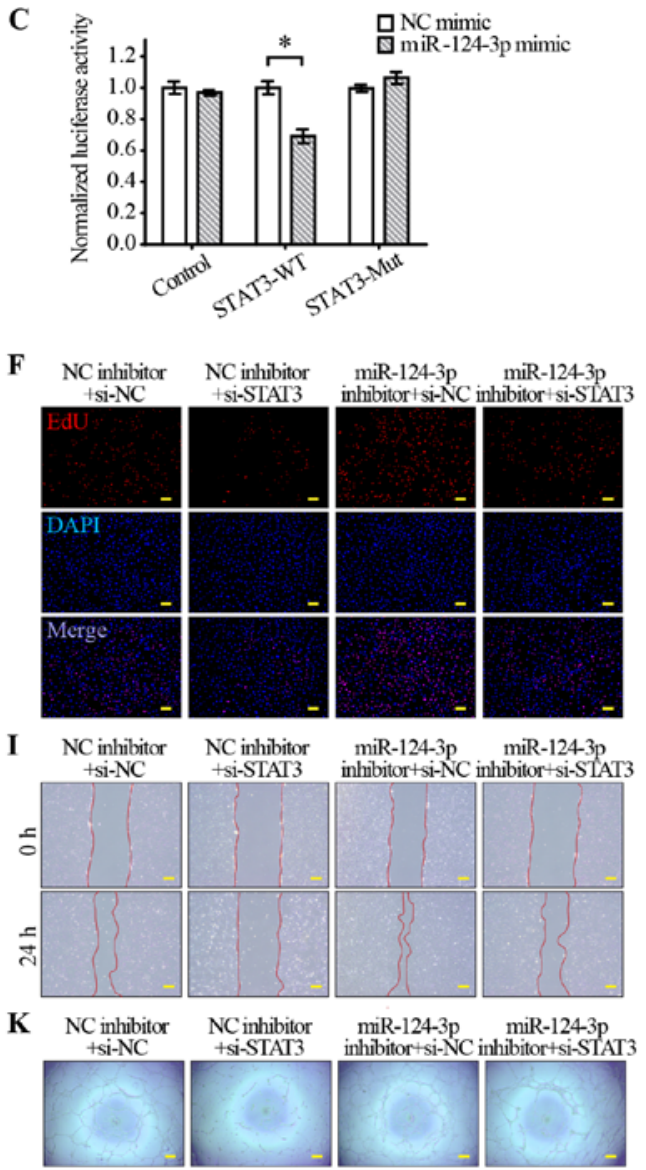

B

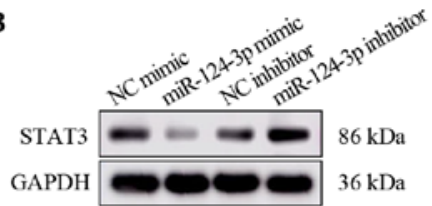

D
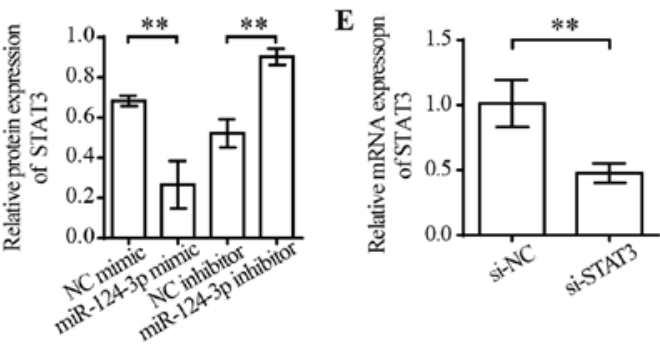

G

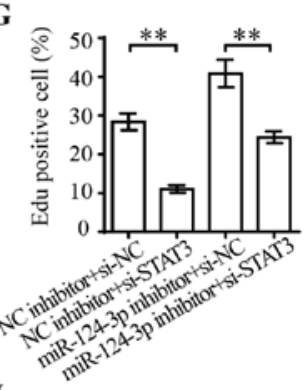

H

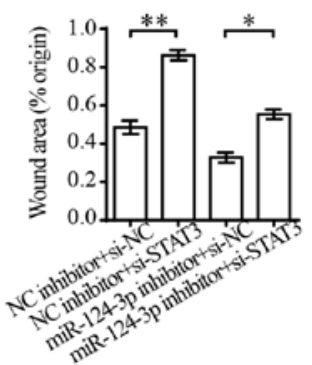

J

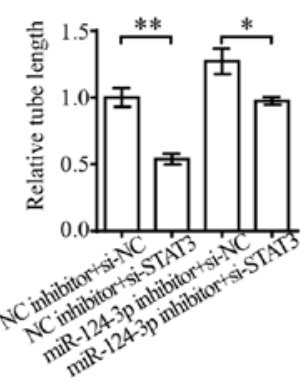

Figure 3. STAT3 is a target of miR-124-3p. (A) Sequence alignment between miR-124-3p and the 3'-UTR of STAT3. (B) Effect of miR-124-3p on STAT3 protein expression in HUVECs. (C) The dual-luciferase reporter assay was performed in 293T cells co-transfected with miR-124-3p mimic or NC mimic and STAT3-WT or STAT3-Mut. (D) Semi-quantified STAT3 protein expression levels in HUVECs transfected with si-STAT3 or si-NC. (E) Transfection efficiency of si-STAT3. HUVEC proliferation was (F) assessed by performing EdU staining (scale bar, $100 \mu \mathrm{m})$ and (G) quantified. (H) HUVEC migration was assessed by performing wound healing assays. (I) Representative images of the wound healing assays. Scale bar, $100 \mu$ m. (J) HUVEC tube formation was assessed by performing tube formation assays. (K) Representative images of the tube formation assays. The experiments were repeated three (A-E) or five $(\mathrm{F}-\mathrm{K})$ times. ${ }^{*} \mathrm{P}<0.05$ and ${ }^{* *} \mathrm{P}<0.01$. miR, microRNA; 3'-UTR, 3 '-untranslated region; HUVECs, human umbilical vein endothelial cells; NC, negative control; WT, wild-type; Mut, mutated; si, small interfering RNA.

miR-124-3p in the tissues and blood of the HLI model displayed a similar peak time, and increased miR-124-3p expression markedly inhibited angiogenesis in ligated legs, suggesting that blood-derived miR-124-3p might serve as a marker of serious PAD. To investigate the hypothesis, 49 patients with PAD and 47 healthy individuals were enrolled in the present study. The baseline characteristics of the patients are presented in Table I. RT-qPCR was conducted to detect the levels of miR-124-3p in human blood. The levels of miR-124-3p in patients with PAD were significantly higher compared with non-PAD individuals (1.85-fold; PAD, $2.03 \pm 0.65$ vs. non-PAD, $1.10 \pm 0.47$; Fig. 5A). ABI is an index for the assessment of the severity of PAD $(31,32)$. Pearson's correlation analysis indicated that the levels of miR-124-3p in the blood were negatively correlated with ABI scores (r=-0.6248; Fig. 5B), suggesting that miR-124-3p expression levels were positively correlated with the severity of PAD.

\section{Discussion}

The concept of therapeutic angiogenesis for PAD has been around for decades, but the advances made thus far fall far below expectations. A potential reason for the lack of effective results from therapeutic angiogenesis could be the complicated self-regulation of cells in the microenvironment $(33,34)$. miRNAs are a type of small molecule that can serve as endogenous regulators of cells $(8,9)$. Although miRNAs have been reported to be widely involved in the regulation of diseases, such as cancers (35), autoimmune diseases (36), central nervous system injuries (37) and heart diseases (38), there is limited information on the involvement of miRNAs in PAD. miR-124-3p was initially reported to be highly expressed in brain tissues (39), serving a critical role in neuronal differentiation (40). Further studies investigated other functions of miR-124-3p. According to Ando et al (15), miR-124-3p 

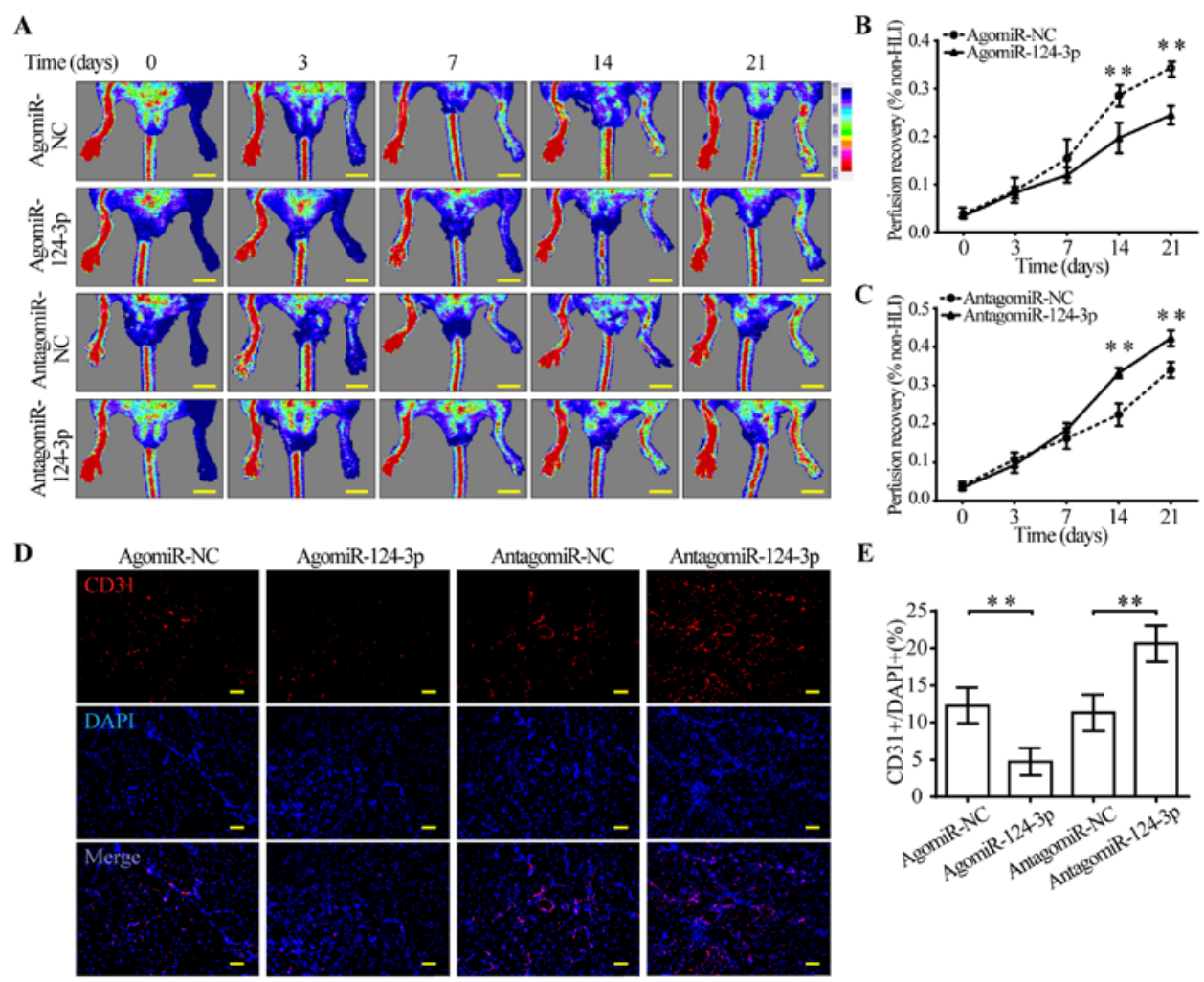

E

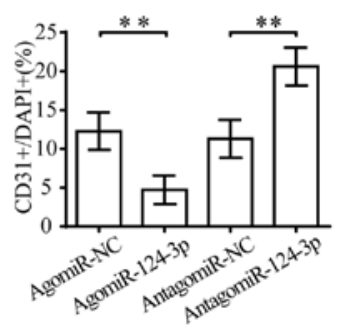

Figure 4. miR-124-3p impairs perfusion recovery and capillary density in the HLI model. miR-124-3p was overexpressed using agomiR-124-3p or knocked down using antagomiR-124-3p in the HLI model. (A) Representative images of perfusion recovery in the HLI model. Dark blue represents low or no perfusion and red represents the highest degree of perfusion. Scale bar, $1 \mathrm{~cm}$. The effects of (B) agomiR-124-3p and (C) antagomiR-124-3p on perfusion recovery were quantified using moorLDI Image Processing software. (D) Representative images of CD31 ${ }^{+}$capillaries of the ischemic gastrocnemius on day 14 post-HLI. Scale bar, $50 \mu \mathrm{m}$. (E) Quantification of the capillary density of gastrocnemius. The experiments were repeated four times. ${ }^{* *} \mathrm{P}<0.01$. miR, microRNA; HLI, hindlimb ischemia.
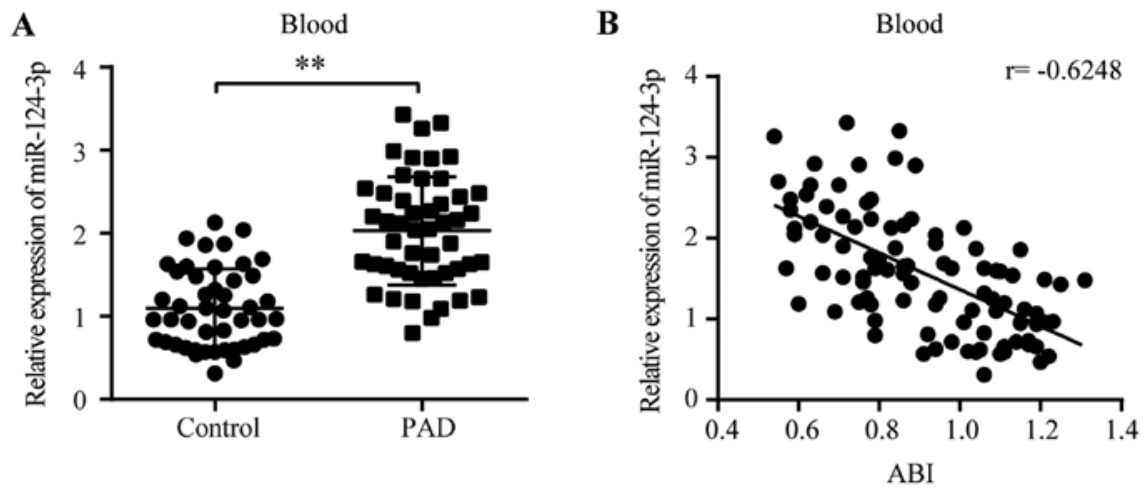

Figure 5. miR-124-3p expression levels are increased in patients with PAD. (A) Relative expression of miR-124-3p in the blood of non-PAD individuals ( $\mathrm{n}=47$ ) and patients with PAD $(n=49)$. (B) Correlation analysis between $A B I$ and the relative expression of miR-124-3p in the blood of patients with PAD and non-PAD individuals $(\mathrm{n}=96 ; \mathrm{r}=-0.6248) .{ }^{* *} \mathrm{P}<0.01$. miR, microRNA; PAD, peripheral arterial disease; $\mathrm{ABI}$, ankle-brachial index.

suppressed tumor development by inhibiting angiogenesis. Shi et al (16) also demonstrated that miR-124-3p might predict acute myocardial infarction, suggesting that miR-124-3p might serve as a regulator of angiogenesis. Therefore, the aforementioned studies highlighted the importance of investigating the correlation between miR-124-3p and angiogenesis in PAD.

In the present study, the results indicated that miR-124-3p was upregulated under hypoxic conditions both in vivo and in vitro compared with the corresponding control groups. Moreover, compared with the NC groups, miR-124-3p overexpression significantly suppressed HUVEC functions and impaired perfusion recovery in the HLI model. STAT3 has been recognized as a regulator of angiogenesis beyond inflammation (41). The functions of STAT3 are precisely regulated by multiple chaperonins under specific conditions. For example, canonical STAT3 signaling is associated with JAK-STAT signaling, whereby STAT3 is phosphorylated on tyrosine 750 (Y750), facilitating STAT3 homodimerization, nuclear translation, DNA binding and initiation of transcription (42). The noncanonical nuclear activities, including acetylation (43), alkylation (44), methylation (45), ubiquitination (46) and glutathionylation (44), have been implicated in STAT3 transcriptional 
activity in various cells. Certain studies have reported the axis of miR-124-3p/STAT3 (47-49), but to the best of our knowledge, no previous study has focused on the functions of STAT3 in EC proliferation. Inhibition of the STAT3 signaling pathway impairs angiogenesis and perfusion recovery in the muscles of patients with PAD (50). In addition, an increasing number of studies have verified that STAT3 was involved in the regulation of tumor angiogenesis by modulating the expression of VEGF $(51,52)$. The results of the present study indicated that miR-124-3p overexpression significantly decreased STAT3 protein expression levels and inhibited HUVEC proliferation compared with NC mimic. Furthermore, it has been reported that STAT3 could bind with Yes-associated protein to regulate the mRNA expression levels of angiopoietin-2 in ECs $(53,54)$. Therefore, the aforementioned results suggested that miR-124-3p regulated angiogenesis following ischemic injury in mouse hindlimbs by targeting STAT3.

Another interesting finding of the present study was that the levels of circulating miR-124-3p were negatively correlated with ABI, the index for PAD severity. ABI is a non-invasive physical index that provides the standard for the evaluation of PAD severity (31). ABI is less sensitive in conditions associated with vessel stiffness (55); therefore, the expression of miR-124-3p in the blood might serve as an improved marker for screening patients than ABI. However, the role of miR-124-3p in the progression of PAD requires further investigation with additional samples. In addition, the long-term outcome of patients with increased miR-124-3p expression requires further investigation.

Although previous studies have reported possible roles of miR-124-3p in ischemic diseases (27-28), there were several novel aspects of the present study. First, the potential role of miR-124-3p was identified in the HLI model. Secondly, the results indicated that the levels of miR-124-3p in human blood were positively correlated with the severity of PAD, which suggested that miR-124-3p might serve as a strong potential target for the evaluation and treatment of PAD. Therefore, the aforementioned findings may aid with the clinical translation of the present study.

In conclusion, the present study provided evidence for the link between miR-124-3p and PAD. miR-124 regulated angiogenesis by decreasing STAT3 expression. Although miRNA-based therapeutics are still being developed, the results of the present study are encouraging and suggested the potential of miR-124 as a diagnostic, prognostic and therapeutic target for PAD in the future.

\section{Acknowledgements}

Not applicable.

\section{Funding}

The present study was supported by the National Natural Science Foundation of China (grant nos. 81670746, 81670230 and 91939101).

\section{Availability of data and materials}

The datasets used and/or analyzed during the current study are available from the corresponding author on reasonable request.

\section{Authors' contributions}

WP and WJ designed the study. YS and XX performed the experiments, analyzed the data and wrote the manuscript. PL, WK, ML, QY, JZ and YX were responsible for collecting blood samples, baseline characteristics of patients and analyzing the data of population study. All authors read and approved the final manuscript, and agreed to be accountable for the work in ensuring that questions related to the integrity of any part of the work were appropriately investigated and resolved.

\section{Ethics approval and consent to participate}

Written informed consent was obtained from all patients. The present study was approved by the Ethics Committee of Shanghai Tenth People's Hospital, Shanghai, China (approval no. 2019-K-153). Animal experiments were approved by the Laboratory Animal Ethics Committee of Shanghai Tenth People's Hospital, Shanghai, China approval no. SHDSYY-2019-2149).

\section{Patient consent for publication}

Not applicable.

\section{Competing interests}

The authors declare that they have no competing interests.

\section{References}

1. Abdulhannan P, Russell DA and Homer-Vanniasinkam S: Peripheral arterial disease: A literature review. Br Med Bull 104: 21-39, 2012

2. Fowkes FG, Rudan D, Rudan I, Aboyans V, Denenberg JO McDermott MM, Norman PE, Sampson UK, Williams LJ, Mensah GA and Criqui MH: Comparison of global estimates of prevalence and risk factors for peripheral artery disease in 2000 and 2010: A systematic review and analysis. Lancet 382: 1329-1340, 2013.

3. Criqui $\mathrm{MH}$ and Aboyans V: Epidemiology of peripheral artery disease. Circ Res 116: 1509-1526, 2015.

4. Collinson DJ and Donnelly R: Therapeutic angiogenesis in peripheral arterial disease: Can biotechnology produce an effective collateral circulation? Eur J Vasc Endovasc Surg 28: 9-23, 2004.

5. Vandekeere S, Dewerchin M and Carmeliet P: Angiogenesis revisited: An overlooked role of endothelial cell metabolism in vessel sprouting. Microcirculation 22: 509-517, 2015.

6. Taniyama Y, Azuma J, Rakugi H and Morishita R: Plasmid DNA-based gene transfer with ultrasound and microbubbles. Curr Gene Ther 11: 485-490, 2011.

7. Forster R, Liew A, Bhattacharya V, Shaw J and Stansby G: Gene therapy for peripheral arterial disease. Cochrane Database Syst Rev 10: CD012058, 2018.

8. Bartel DP: MicroRNAs: Target recognition and regulatory functions. Cell 136: 215-233, 2009.

9. Lu TX and Rothenberg ME: MicroRNA. J Allergy Clin Immunol 141: 1202-1207, 2018.

10. Bartel DP: Metazoan MicroRNAs. Cell 173: 20-51, 2018.

11. miRBase: Release 22.1. http://www.mirbase.org. Accessed October 2018.

12. Zhao J, Li X, Hu J, Chen F, Qiao S, Sun X, Gao L, Xie J and Xu B: Mesenchymal stromal cell-derived exosomes attenuate myocardial ischaemia-reperfusion injury through miR-182-regulated macrophage polarization. Cardiovasc Res 115: 1205-1216, 2019.

13. Hou Z, Qin X, Hu Y, Zhang X, Li G, Wu J, Li J, Sha J, Chen J, Xia J, et al: Longterm exercise-derived exosomal miR-342-5p: A novel exerkine for cardioprotection. Circ Res 124: 1386-1400, 2019. 
14. Xu X, Tian L and Zhang Z: Triptolide inhibits angiogenesis in microvascular endothelial cells through regulation of miR-92a. J Physiol Biochem 75: 573-583, 2019.

15. Ando H, Okamoto A, Yokota M, Shimizu K, Asai T, Dewa T and Oku N: Development of a miR-92a delivery system for anti-angiogenesis-based cancer therapy. J Gene Med 15: 20-27, 2013.

16. Shi Z, Chen Q, Li C, Wang L, Qian X, Jiang C, Liu X, Wang X, $\mathrm{Li} \mathrm{H}$, Kang C, et al: MiR-124 governs glioma growth and angiogenesis and enhances chemosensitivity by targeting R-Ras and N-Ras. Neuro Oncol 16: 1341-1353, 2014.

17. Zhang G, Chen L, Khan AA, Li B, Gu B, Lin F, Su X and Yan J: miRNA-124-3p/neuropilin-1(NRP-1) axis plays an important role in mediating glioblastoma growth and angiogenesis. Int J Cancer 143: 635-644, 2018

18. Guo ML, Guo LL and Weng YQ: Implication of peripheral blood miRNA-124 in predicting acute myocardial infarction. Eur Rev Med Pharmacol Sci 21: 1054-1059, 2017.

19. Xu SY, Jiang XL, Liu Q, Xu J, Huang J, Gan SW, Lu WT, Zhuo F, Yang $M$ and Sun SQ: Role of rno-miR-124-3p in regulating MCT1 expression in rat brain after permanent focal cerebral ischemia. Genes Dis 6: 398-406, 2019.

20. Livak KJ and Schmittgen TD: Analysis of relative gene expression data using real-time quantitative PCR and the 2(-Delta Delta C(T)) method. Methods 25: 402-408, 2001

21. He Y, Luo Y, Tang S, Rajantie I, Salven P, Heil M, Zhang R, Luo D, Li X, Chi H, et al: Critical function of Bmx/Etk in ischemia-mediated arteriogenesis and angiogenesis. J Clin Invest 116: 2344-2355, 2006.

22. Hazarika S, Farber CR, Dokun AO, Pitsillides AN, Wang T, Lye RJ and Annex BH: MicroRNA-93 controls perfusion recovery after hindlimb ischemia by modulating expression of multiple genes in the cell cycle pathway. Circulation 127: 1818-1828, 2013.

23. National Reaearch Council (US) Committee for the Guide for the Care and Use of Laboratory Animals: Guide for the Care and Use of Laboratory Animals. 8th edition. National Academies Press (US), Washington, DC, 2011.

24. Gerhard-Herman MD, Gornik HL, Barrett C, Barshes NR Corriere MA, Drachman DE, Fleisher LA, Fowkes FGR, Hamburg NM, Kinlay S, et al: 2016 AHA/ACC Guideline on the Management of Patients With Lower Extremity Peripheral Artery Disease: A Report of the American College of Cardiology/American Heart Association Task Force on Clinical Practice Guidelines. J Am Coll Cardiol 69: e71-e126, 2017.

25. Aboyans V, Criqui MH, Abraham P, Allison MA, Creager MA, Diehm C, Fowkes FG, Hiatt WR, Jönsson B, Lacroix P, et al: Measurement and interpretation of the ankle-brachial index: A scientific statement from the American Heart Association. Circulation 126: 2890-2909, 2012.

26. Wu D, Potluri N, Lu J, Kim Y and Rastinejad F: Structural integration in hypoxia-inducible factors. Nature 524: 303-308, 2015.

27. Semenza GL: Life with oxygen. Science 318: 62-64, 2007.

28. Muñoz-Sánchez $\mathrm{J}$ and Chánez-Cárdenas ME: The use of cobalt chloride as a chemical hypoxia model. J Appl Toxicol 39: 556-570, 2019.

29. Chung AS and Ferrara N: Developmental and pathological angiogenesis. Annu Rev Cell Dev Biol 27: 563-584, 2011.

30. Miyazaki T, Taketomi Y, Saito Y, Hosono T, Lei XF, Kim-Kaneyama J, Arata S, Takahashi H, Murakami M and Miyazaki A: Calpastatin counteracts pathological angiogenesis by inhibiting suppressor of cytokine signaling 3 degradation in vascular endothelial cells. Circ Res 116: 1170-1181, 2015

31. Lin JS, Olson CM, Johnson ES and Whitlock EP: The ankle-brachial index for peripheral artery disease screening and cardiovascular disease prediction among asymptomatic adults: A systematic evidence review for the U.S. Preventive Services Task Force. Ann Intern Med 159: 333-341, 2013.

32. Kravos A and Bubnic-Sotosek K: Ankle-brachial index screening for peripheral artery disease in asymptomatic patients between 50 and 70 years of age. J Int Med Res 37: 1611-1619, 2009.

33. Veith AP, Henderson K, Spencer A, Sligar AD and Baker AB: Therapeutic strategies for enhancing angiogenesis in wound healing. Adv Drug Deliv Rev 146: 97-125, 2019.

34. Mitsos S, Katsanos K, Koletsis E, Kagadis GC, Anastasiou N, Diamantopoulos A, Karnabatidis D and Dougenis D: Therapeutic angiogenesis for myocardial ischemia revisited: Basic biological concepts and focus on latest clinical trials. Angiogenesis 15 $1-22,2012$.
35. Rupaimoole R, Calin GA, Lopez-Berestein G and Sood AK: miRNA deregulation in cancer cells and the tumor microenvironment. Cancer Discov 6: 235-246, 2016.

36. Mehta A and Baltimore D: MicroRNAs as regulatory elements in immune system logic. Nat Rev Immunol 16: 279-294, 2016.

37. Bhalala OG, Srikanth M and Kessler JA: The emerging roles of microRNAs in CNS injuries. Nat Rev Neurol 9: 328-339, 2013.

38. Katz MG, Fargnoli AS, Kendle AP, Hajjar RJ and Bridges CR: The role of microRNAs in cardiac development and regenerative capacity. Am J Physiol Heart Circ Physiol 310: H528-541, 2016.

39. Sempere LF, Freemantle S, Pitha-Rowe I, Moss E, Dmitrovsky E and Ambros V: Expression profiling of mammalian microRNAs uncovers a subset of brain-expressed microRNAs with possible roles in murine and human neuronal differentiation. Genome Biol 5: R13, 2004.

40. Yoo AS, Sun AX, Li L, Shcheglovitov A, Portmann T, Li Y, Lee-Messer C, Dolmetsch RE, Tsien RW and Crabtree GR: MicroRNA-mediated conversion of human fibroblasts to neurons. Nature 476: 228-231, 2011.

41. Chen Z and Han ZC: STAT3: A critical transcription activator in angiogenesis. Med Res Rev 28: 185-200, 2008.

42. Koo MY, Park J, Lim JM, Joo SY, Shin SP, Shim HB, Chung J, Kang D, Woo HA and Rhee SG: Selective inhibition of the function of tyrosine-phosphorylated STAT3 with a phosphorylation site-specific intrabody. Proc Natl Acad Sci USA 111: 6269-6274, 2014.

43. Yuan ZL, Guan YJ, Chatterjee D and Chin YE: Stat3 dimerization regulated by reversible acetylation of a single lysine residue. Science 307: 269-273, 2005.

44. Buettner R, Corzano R, Rashid R, Lin J, Senthil M, Hedvat M, Schroeder A, Mao A, Herrmann A, Yim J, et al: Alkylation of cysteine 468 in Stat 3 defines a novel site for therapeutic development. ACS Chem Biol 6: 432-443, 2011.

45. Stark GR, Kerr IM, Williams BR, Silverman RH and Schreiber RD: How cells respond to interferons. Annu Rev Biochem 67: 227-264, 1998.

46. Stark GR, Wang Y and Lu T: Lysine methylation of promoter-bound transcription factors and relevance to cancer. Cell Res 21: 375-380, 2011.

47. Zhou YL, Zhang L, Zhou Z, Liu W, Lu Y, He S, Cui Y, Qin Y and Hua M: Antibody modified nanoparticle-mediated delivery of miR-124 regulates apoptosis via repression the Stat3 signal in mycobacterial-infected microglia. J Biomed Nanotechnol 14: 2185-2197, 2018.

48. Zhang Y, Li X, Zhang J and Liang H: Natural killer T cell cytotoxic activity in cervical cancer is facilitated by the LINC00240/microRNA-124-3p/STAT3/MICA axis. Cancer Lett 474: 63-73, 2020.

49. Vuokila N, Aronica E, Korotkov A, van Vliet EA, Nuzhat S, Puhakka N and Pitkanen A: Chronic regulation of miR-124-3p in the perilesional cortex after experimental and human TBI. Int J Mol Sci 21: 2418, 2020.

50. Ganta VC, Choi M, Kutateladze A and Annex BH: VEGF165b modulates endothelial VEGFR1-STAT3 signaling pathway and angiogenesis in human and experimental peripheral arterial disease. Circ Res 120: 282-295, 2017.

51. Banerjee $\mathrm{K}$ and Resat $\mathrm{H}$ : Constitutive activation of STAT3 in breast cancer cells: A review. Int J Cancer 138: 2570-2578, 2016.

52. Tartour E, Pere H, Maillere B, Terme M, Merillon N, Taieb J, Sandoval F, Quintin-Colonna F, Lacerda K, Karadimou A, et al: Angiogenesis and immunity: A bidirectional link potentially relevant for the monitoring of antiangiogenic therapy and the development of novel therapeutic combination with immunotherapy. Cancer Metastasis Rev 30: 83-95, 2011.

53. He J, Bao Q, Zhang Y, Liu M, Lv H, Liu Y, Yao L, Li B, Zhang C, $\mathrm{He} \mathrm{S}$, et al: Yes-associated protein promotes angiogenesis via signal transducer and activator of transcription 3 in endothelial cells. Circ Res 122: 591-605, 2018.

54. Wan L, Zhang Q, Wang S, Gao Y, Chen X, Zhao Y and Qian X: Gambogic acid impairs tumor angiogenesis by targeting YAP/STAT3 signaling axis. Phytother Res 33: 1579-1591, 2019.

55. Suominen V, Uurto I, Saarinen J, Venermo M and Salenius J: PAD as a risk factor for mortality among patients with elevated ABI-A clinical study. Eur J Vasc Endovasc Surg 39: 316-322, 2010.

This work is licensed under a Creative Commons Attribution-NonCommercial-NoDerivatives 4.0 International (CC BY-NC-ND 4.0) License. 